\title{
An Introduction to Convex Optimization for Communications and Signal Processing
}

\author{
Zhi-Quan Luo, Senior Member, IEEE, and Wei Yu, Member, IEEE
}

Tutorial Paper

\begin{abstract}
Convex optimization methods are widely used in the design and analysis of communication systems and signal processing algorithms. This tutorial surveys some of recent progress in this area. The tutorial contains two parts. The first part gives a survey of basic concepts and main techniques in convex optimization. Special emphasis is placed on a class of conic optimization problems, including second-order cone programming and semidefinite programming. The second half of the survey gives several examples of the application of conic programming to communication problems. We give an interpretation of Lagrangian duality in a multiuser multi-antenna communication problem; we illustrate the role of semidefinite relaxation in multiuser detection problems; we review methods to formulate robust optimization problems via second-order cone programming techniques.
\end{abstract}

Index Terms-Convex optimization, digital communications, duality, second-order cone programming (SOCP), semidefinite programming (SDP), signal processing.

\section{INTRODUCTION}

$\mathbf{T}$ HE USE OF optimization methods is ubiquitous in communications and signal processing. Many communication problems can either be cast as or be converted into convex optimization problems, which greatly facilitate their analytic and numerical solutions. This tutorial paper gives a survey of some of recent progress in convex optimization techniques for digital communications and signal processing applications.

Convex optimization refers to the minimization of a convex objective function subject to convex constraints. Convex optimization techniques are important in engineering applications because a local optimum is also a global optimum in a convex problem and a rigorous optimality condition and a duality theory exist to verify the optimal solution. Consequently, when a design problem is cast into a convex form, the structure of the optimal solution, which often reveals design insights, can often be identified. Further, powerful numerical algorithms exist to solve for the optimal solution of convex problems efficiently.

Manuscript received May 3, 2006; revised May 8, 2006. The work of Z.-Q. Luo was supported in part by the National Science Foundation under Grant DMS-0312416. The work of W. Yu was supported in part by the Natural Sciences and Engineering Research Council (NSERC) of Canada and in part by the Canada Research Chairs Program.

Z.-Q. Luo is with the Department of Electrical and Computer Engineering, University of Minnesota, Minneapolis, MN 55455 USA (e-mail: luozq@ece. umn.edu).

W. Yu is with The Edward S. Rogers Sr. Department Electrical and Computer Engineering, University of Toronto, Toronto, ON M5S 3G4, Canada (e-mail: weiyu@comm.utoronto.ca).

Digital Object Identifier 10.1109/JSAC.2006.879347
There have been significant advances in the research in convex optimization (e.g., interior-point method [1] and conic optimization [2]) over the last two decades. The first part of this tutorial provides an overview of these developments and describes the basic optimization concepts, models and tools that are most relevant to signal processing and communication applications. The second half of this paper includes examples illustrating Lagrangian duality, the method of relaxation, and robust optimization techniques, which are useful in many engineering applications. Open problems and future research directions are also discussed.

\section{CONVEX OPTIMIZATION}

In order to recognize convex optimization problems in engineering applications, one must first be familiar with the basic concepts of convexity and the commonly used convex optimization models. This section provides a concise review of these optimization concepts and models including linear programming, second-order cone programming, and semidefinite cone programming, all illustrated through concrete examples. In addition, the Karush-Kuhn-Tucker optimality conditions are reviewed and stated explicitly for each of the convex optimization models, followed by a description of the well-known interior-point algorithms and a brief discussion of their worst-case complexity.

Here and throughout, vectors are in lower case letters, and matrices are in capital case letters. The transpose is expressed by $(\cdot)^{T}$; the conjugate transpose is expressed by $(\cdot)^{H}$; the trace is expressed by $\operatorname{tr}(\cdot)$. The set of $n$ by $n$ positive semidefinite real symmetric matrices is denoted by $\mathcal{S}_{+}^{n}$; the set of $n$ by $n$ positive semidefinite complex Hermitian matrices is denoted by $\mathcal{H}_{+}^{n}$. For two given matrices $A$ and $B$, we use " $A \succeq B$ " to indicate that $A-B$ is positive semidefinite, and $A \bullet B:=$ $\sum_{i, j} A_{i j} B_{i j}=\operatorname{tr}\left(A B^{T}\right)$ to denote the matrix inner product. The Frobenius norm of $A$ is denoted by $\|A\|_{F}=\sqrt{\operatorname{tr}\left(A A^{T}\right)}$. The Euclidean norm of a vector $x \in \Re^{n}$ is denoted as $\|x\|$.

\section{A. Basic Optimization Concepts}

Convex Sets: A set $S \subset \Re^{n}$ is said to be convex if for any two points $x, y \in S$, the line segment joining $x$ and $y$ also lies in $S$. Mathematically, it is defined by the following property:

$$
\theta x+(1-\theta) y \in S, \quad \forall \theta \in[0,1] \text { and } x, y \in S .
$$

Many well-known sets are convex, for example, the unit ball $S=\{x \mid\|x\| \leq 1\}$. However, the unit sphere $S=\{x \mid\|x\|=$ 
$1\}$ is not convex since the line segment joining any two distinct points is no longer on the unit sphere. In general, a convex set must be a solid body, containing no holes, and always curve outward. Other examples of convex sets include ellipsoids, hypercubes, polyhedral sets, and so on. In the real line $\Re$, convex sets correspond to intervals (open or closed). The most important property about convex set is the fact that the intersection of any number (possibly uncountable) of convex sets remains convex. For example, the set $S=\{x \mid\|x\| \leq 1, x \geq 0\}$ is the intersection of the unit ball with the nonnegative orthant $\left(\Re_{+}^{n}\right)$, both of which are convex. Thus, their intersection $S$ is also convex. The union of two convex sets is typically nonconvex.

Convex Cones: A convex cone $\mathcal{K}$ is a special type of convex set which is closed under positive scaling: for each $x \in \mathcal{K}$ and each $\alpha \geq 0, \alpha x \in \mathcal{K}$. Convex cones arise in various forms in engineering applications. The most common convex cones are the following.

1) Nonnegative orthant $\Re_{+}^{n}$.

2) Second-order cone (also known as ice-cream cone)

$$
\mathcal{K}=\operatorname{SOC}(n)=\{(t, x) \mid t \geq\|x\|\} .
$$

In practice, sometimes it is more convenient to work with the so-called rotated second-order cone: $\left\{(t, s, x) \in \Re^{n} \mid t s \geq\|x\|^{2}, t \geq 0, s \geq 0\right\}$. This cone is equivalent to the standard $\operatorname{SOC}(n+1)$ via a simple linear transformation.

3) Positive semidefinite matrix cone

$$
\mathcal{K}=\mathcal{S}_{+}^{n}=\{X \mid X \text { symmetric and } X \succeq 0\} .
$$

For any convex cone $\mathcal{K}$, we can define its dual cone

$$
\mathcal{K}^{*}=\{x \mid\langle x, y\rangle \geq 0, \forall y \in \mathcal{K}\}
$$

where $\langle\cdot, \cdot\rangle$ denotes the inner product operation. In other words, the dual cone $\mathcal{K}^{*}$ consists of all vectors $y$ which forms a nonobtuse angle with all vectors in $\mathcal{K}$. We say $\mathcal{K}$ is self-dual if $\mathcal{K}=\mathcal{K}^{*}$. It can be shown that the nonnegative orthant cone, the second-order cone and the symmetric positive semidefinite matrix cone are all self-dual. Notice that for the second-order cone, the inner product operation $\langle\cdot, \cdot\rangle$ is defined as

$$
\begin{aligned}
& \langle(t, x),(s, y)\rangle=t s+x^{T} y, \\
& \forall(t, x) \text { and }(s, y) \text { with } t \geq\|x\| \text { and } s \geq\|y\|
\end{aligned}
$$

and for the positive semidefinite matrix cone

$$
\langle X, Y\rangle=X \bullet Y=\sum_{i, j} X_{i j} Y_{i j}
$$

Convex Functions: A function $f(x): \Re^{n} \rightarrow \Re$ is said to be convex if for any two points $x, y \in \Re^{n}$

$$
f(\theta x+(1-\theta) y) \leq \theta f(x)+(1-\theta) f(y), \quad \forall \theta \in[0,1] .
$$

Geometrically, this means that, when restricted over the line segment joining $x$ and $y$, the linear function joining $(x, f(x))$ and $(y, f(y))$ always dominates the function $f$. There are many examples of convex functions, including the commonly seen univariate functions $|x|, e^{x}, x^{2}$, as well as multivariate functions $a^{T} x+b,\|A x\|^{2}$, where $A, a$, and $b$ are given data matrix/vector/constant. We say $f$ is concave if $-f$ is convex. The entropy function $-\sum_{i} x_{i} \log x_{i}$ is a concave function over $\Re_{+}^{n}$. If $f$ is continuously differentiable, then the convexity of $f$ is equivalent to

$$
f(y) \geq f(x)+\nabla f(x)^{T}(y-x), \quad \forall x, y \in \Re^{n} .
$$

In other words, the first-order Taylor series expansion serves as a global underestimator of $f$. Furthermore, if $f$ is twice continuously differentiable, then the convexity of $f$ is equivalent to the positive semidefiniteness of its Hessian: $\nabla^{2} f(x) \succeq 0$, $\forall x \in \Re^{n}$. Thus, a linear function is always convex, while a quadratic function $x^{T} P x+a^{T} x+b$ is convex if and only if $P \succeq 0$. Notice that the linear plus the constant term $a^{T} x+b$ does not have any bearing on the convexity (or the lack of) of $f$. One can think of numerous examples of functions which are neither convex nor concave. For instance, the function $x^{3}$ is convex over $[0, \infty)$ and concave over the region $(-\infty, 0]$, but is neither convex nor concave over $\Re$.

The most important property about convex functions is the fact that they are closed under summation, positive scaling, and the pointwise maximum operations. In particular, if the $\left\{f_{i}\right\}$ 's are convex, then so is $\max _{i}\left\{f_{i}(x)\right\}$ (even though it is typically nondifferentiable). A notable connection between convex set and convex function is the fact that the level sets of any convex function $f(x)$ are always convex, i.e., $\{x \mid f(x) \leq c\}$ is convex for any $c \in \Re$. The converse is not true, however. For example, the function $f(x)=\sqrt{|x|}$ is nonconvex, but its level sets are convex.

Convex Optimization Problems: Consider a generic optimization problem (in the minimization form)

$$
\begin{array}{ll}
\text { minimize } & f_{0}(x) \\
\text { subject to } & f_{i}(x) \leq 0, i=1,2, \ldots, m, \\
& h_{j}(x)=0, j=1,2, \ldots, r, \\
& x \in S
\end{array}
$$

where $f_{0}$ is called the objective function (or cost function), $\left\{f_{i}\right\}_{i=1}^{m}$ and $\left\{h_{j}\right\}_{j=1}^{r}$ are called the inequality and equality constraint functions, respectively, and $S$ is called a constraint set. In practice, $S$ can be implicitly defined by an oracle such as a user-supplied software. The optimization variable $x \in \Re^{n}$ is said to be feasible if $x \in S$ and it satisfies all the inequality and equality constraints. A feasible solution $x^{*}$ is said to be globally optimal if $f_{0}\left(x^{*}\right) \leq f_{0}(x)$ for all feasible $x$. In contrast, a feasible vector $\bar{x}$ is said to be locally optimal if there exists some $\epsilon>0$ such that $f_{0}(\bar{x}) \leq f_{0}(x)$ for all feasible $x$ satisfying $\|x-\bar{x}\| \leq \epsilon$.

The optimization problem (2) is said to be convex if 1) the functions $f_{i}(i=0,1,2, \ldots m)$ are convex; 2$) h_{j}(x)$ are affine functions (i.e., $h_{j}$ is of the form $a_{j}^{T} x+b_{j}$ for some $a_{j} \in \Re^{n}$ and $b_{j} \in \Re$ ); and 3 ) the set $S$ is convex. Violating any one of the three conditions will result in a nonconvex problem. Notice that if we change "minimize" to "maximize" and change direction of the inequalities from " $f_{i}(x) \leq 0$ " to " $f_{i}(x) \geq 0$," then (2) is convex if and only if all $f_{i}(x)(i=0,1,2, \ldots, m)$ are concave. 
For example, the following entropy maximization problem is convex:

$$
\begin{aligned}
\text { maximize } & \sum_{i=1}^{n} x_{i} \log x_{i} \\
\text { subject to } & \sum_{i=1}^{n} x_{i}=1, x_{i} \geq 0, i=1,2, \ldots, n, \\
& A x=b, j=1,2, \ldots, r
\end{aligned}
$$

where the linear equalities $A x=b$ may represent the usual moment matching constraints.

Let us now put in perspective the role of convexity in optimization. It is well known that, for the problem of solving a system of equations, linearity is the dividing line between the "easy" and "difficult" problems. ${ }^{1}$ Once a problem is formulated as a solution to a system of linear equations, the problem is considered done since we can simply solve it either analytically or using existing numerical softwares. In fact, there are many efficient and reliable softwares available for solving systems of linear equations, but none for nonlinear equations. The lack of high-quality softwares for solving nonlinear equations is merely a reflection of the fact that they are intrinsically difficult to solve.

In contrast, the dividing line between the "easy" and "difficult" problems in optimization is no longer linearity, but rather convexity. Convex optimization problems are the largest subclass of optimization problems which are efficiently solvable, whereas nonconvex optimization problems are generally difficult. The theory, algorithms and software tools for convex optimization problems have advanced significantly over the last 50 years. There are now (freely downloadable) high-quality softwares which can deliver accurate solutions efficiently and reliably without the usual headaches of initialization, step-size selection or the risk of getting trapped in a local minimum. Once an engineering problem is formulated in a convex manner, it is reasonable to consider it "solved" (or "game over"), at least from a numerical perspective.

For any convex optimization problem, the set of global optimal solutions is always convex. Moreover, every local optimal solution is also a global optimal solution, so there is no danger of being stuck at a local solution. There are other benefits associated with a convex optimization formulation. For one thing, there exist highly efficient interior-point optimization algorithms whose worst-case complexity (i.e., the total number of arithmetic operations required to find an $\epsilon$-optimal solution) grows gracefully as a polynomial function of the problem data length and $\log 1 / \epsilon$. In addition, there exists an extensive duality theory for convex optimization problems, a consequence of which is the existence of a computable mathematical certificate for infeasible convex optimization problems. As a result, well-designed softwares for solving convex optimization problems typically return either an optimal solution, or a certificate (in the form of a dual vector) that establishes the infeasibility of the problem. The latter property is extremely valuable in engineering design since it enables us to identify constraints which are too restrictive.

\footnotetext{
${ }^{1}$ These notions can be made precise using the computational complexity theory; e.g., NP-hardness results.
}

\section{B. Lagrangian Duality and Karush-Kuhn-Tucker Condition}

Consider the following (not necessarily convex) optimization problem:

$$
\begin{array}{ll}
\operatorname{minimize} & f_{0}(x) \\
\text { subject to } & f_{i}(x) \leq 0, i=1,2, \ldots, m, \\
& h_{j}(x)=0, j=1,2, \ldots, r, \\
& x \in S .
\end{array}
$$

Let $p^{*}$ denote the global minimum value of (3). For symmetry reason, we will call (3) the primal optimization problem, and call $x$ the primal vector. Introducing dual variables $\lambda \in \Re^{m}$ and $\nu \in \Re^{r}$, we can form the Lagrangian function

$$
L(x, \lambda, \nu):=f_{0}(x)+\sum_{i=1}^{m} \lambda_{i} f_{i}(x)+\sum_{j=1}^{r} \nu_{j} h_{j}(x) .
$$

The so-called dual function $g(\lambda, \nu)$ associated with (3) is defined as

$$
g(\lambda, \nu):=\min _{x \in S} L(x, \lambda, \nu) .
$$

Notice that, as a pointwise minimum of a family of linear functions (in $(\lambda, \nu)$ ), the dual function $g(\lambda, \nu)$ is always concave. We will say $(\lambda, \nu)$ is dual feasible if $\lambda \geq 0$ and $g(\lambda, \nu)$ is finite. The well-known weak duality result says the following.

Proposition 1: For any primal feasible vector $x$ and any dual feasible vector $(\lambda, \nu)$, there holds

$$
f_{0}(x) \geq g(\lambda, \nu) .
$$

In other words, for any dual feasible vector $(\lambda, \nu)$, the dual function value $g(\lambda, \nu)$ always serves as a lower bound on the primal objective value $f_{0}(x)$. Notice that $x$ and $(\lambda, \nu)$ are chosen independent from each other (so long as they are both feasible). Thus, $p^{*} \geq g(\lambda, \nu)$ for all dual feasible vector $(\lambda, \nu)$. The largest lower bound for $p^{*}$ can be found by solving the following dual optimization problem:

$$
\begin{array}{ll}
\text { maximize } & g(\lambda, \nu) \\
\text { subject to } & \lambda \geq 0, \nu \in \Re^{r} .
\end{array}
$$

Notice that the dual problem (4) is always convex regardless of the convexity of the primal problem (3), since $g(\lambda, \nu)$ is concave. Let us denote the maximum value of (4) by $d^{*}$. Then, we have $p^{*} \geq d^{*}$. Interestingly, for most convex optimization problems (satisfying some mild constraint qualification conditions, such as the existence of a strict interior point), we actually have $p^{*}=d^{*}$. This is called strong duality.

In general, the dual function $g(\lambda, \nu)$ is difficult to compute. However, for special classes of convex optimization problems (see Section II-C), we can derive their duals explicitly. Below is a simple example illustrating the concept of duality for linear programming.

Example: Let us consider the following linear programming problem:

$$
\begin{array}{ll}
\operatorname{minimize} & x_{1}+x_{2} \\
\text { subject to } & x_{1}+2 x_{2}=2, \\
& \left(x_{1}, x_{2}\right)^{T} \in \Re_{+}^{2} .
\end{array}
$$


The primal optimal solution is unique and equal to $\left(x_{1}^{*}, x_{2}^{*}\right)=$ $(0,1)$, with $p^{*}=x_{1}^{*}+x_{2}^{*}=1$. The Lagrangian function is given by $L(x, \nu)=x_{1}+x_{2}+\nu\left(2-x_{1}-2 x_{2}\right)$, and the dual function is given by

$$
\begin{aligned}
g(\nu) & =\min _{\left(x_{1}, x_{2}\right)^{T} \in \Re_{+}^{2}}\left\{x_{1}+x_{2}+\nu\left(2-x_{1}-2 x_{2}\right)\right\} \\
& =2 \nu+\min _{\left(x_{1}, x_{2}\right)^{T} \in \Re_{+}^{2}}\left\{(1-\nu) x_{1}+(1-2 \nu) x_{2}\right\} \\
& =\left\{\begin{array}{ll}
2 \nu, & \text { if } \nu \leq \frac{1}{2} \\
-\infty, & \text { otherwise }
\end{array} .\right.
\end{aligned}
$$

Thus, the dual linear program can be written as

$$
\begin{array}{ll}
\operatorname{maximize} & 2 \nu \\
\text { subject to } & \nu \leq \frac{1}{2}
\end{array}
$$

Clearly, the dual optimal solution is given by $\nu^{*}=1 / 2$ and the dual optimal objective value is $d^{*}=1$. Thus, we have in this case $p^{*}=d^{*}$. In light of Proposition 1, the dual optimal solution $\nu^{*}=1 / 2$ serves as a certificate for the primal optimality of $\left(x_{1}^{*}, x_{2}^{*}\right)$.

Next, we present a local optimality condition for the optimization problem (3). For ease of exposition, let us assume $S=\Re$. Then, a necessary condition for $x^{*}$ to be a local optimal solution of (3) is that there exists some $\left(\lambda^{*}, \nu^{*}\right)$ such that

and

$$
\begin{aligned}
f_{i}\left(x^{*}\right) & \leq 0, \quad \forall i=1,2, \ldots, m \\
h_{j}\left(x^{*}\right) & =0, \quad \forall j=1,2, \ldots, r \\
\lambda^{*} & \geq 0, \\
\lambda_{i}^{*} f_{i}\left(x^{*}\right) & =0, \quad \forall i=1,2, \ldots, m
\end{aligned}
$$

$$
\nabla f_{0}\left(x^{*}\right)+\sum_{i=1}^{m} \lambda_{i}^{*} \nabla f_{i}\left(x^{*}\right)+\sum_{j=1}^{r} \nu_{j}^{*} \nabla h_{j}\left(x^{*}\right)=0 .
$$

Collectively, the conditions (6)-(10) are called the KarushKuhn-Tucker (KKT) condition for optimality. Notice that the first two conditions (6) and (7) represent primal feasibility of $x^{*}$, condition (8) represents dual feasibility, condition (9) signifies the complementary slackness for the primal and dual inequality constraint pairs: $f_{i}(x) \leq 0$ and $\lambda_{i} \geq 0$, while the last condition (10) is equivalent to $\nabla_{x} L\left(x^{*}, \lambda^{*}, \nu^{*}\right)=0$.

For the above linear programming example, we can easily check that the vector $\left(x_{1}^{*}, x_{2}^{*}\right)=(0,1)$ and the Lagrangian multipliers $\left(\lambda_{1}^{*}, \lambda_{2}^{*}, \nu^{*}\right)=(1 / 2,0,1 / 2)$ satisfy the above KKT condition. Moreover, they are the unique solution of (6)-(10). Thus, $\left(x_{1}^{*}, x_{2}^{*}\right)=(0,1)$ is the unique primal optimal solution for (5).

In general, the KKT condition is necessary but not sufficient for optimality. However, for convex optimization problems (and under mild constraint qualification conditions), the KKT condition is also sufficient. If the constraints in (3) are absent, the corresponding KKT condition simply reduces to the well-known stationarity condition for unconstrained optimization problem: $\nabla f_{0}\left(x^{*}\right)=0$. That is, an unconstrained local minimum must be attained at a stationary point (at which the gradient of $f_{0}$ vanishes). However, in the presence of constraints, local optimal solution of (3) is no longer attained at a stationary point; instead, it is attained at a KKT point $x^{*}$, which, together with some dual feasible vector $\left(\lambda^{*}, \nu^{*}\right)$, satisfies the KKT condition (6)-(10).

Detecting Infeasibility: Efficient detection of infeasibility is essential in engineering design applications. However, the problem of detecting and removing the incompatible constraints is NP-hard in general, especially if the constraints are nonconvex. However, for convex constraints, we can make use of duality theory to prove inconsistency. Let us consider the following example.

Example: Determine if the following linear system is feasible:

$$
\begin{aligned}
x_{1}+x_{2} & \leq 1 \\
x_{1}-x_{2} & \leq-1 \\
-x_{1} & \leq-1 .
\end{aligned}
$$

Let us multiply the last inequality by 2 and add it to the first and the second inequalities. The resulting inequality is $0 \leq-1$, which is a contradiction. This shows that the above linear system is infeasible.

In general, a linear system of inequalities

$$
A x \leq b
$$

is infeasible if and only if there exists some $\lambda \geq 0$, such that

$$
\lambda^{T} A=0, \quad \lambda^{T} b<0
$$

Clearly, the existence of a such $\lambda$ serves as a certificate for the incompatibility of the linear inequalities in (11). What is interesting (and nontrivial) is the fact that the converse is also true. That is, if the system (11) is infeasible, then there always exists a mathematical certificate $\lambda$ satisfying (12). Results of this kind are called the theorems of alternatives, and are related to the well-known Farkas' lemma for the linear feasibility problem.

The above result can also be extended to the nonlinear context. For instance, consider a system of convex (possibly nonlinear) inequality system

$$
f_{1}(x)<0, f_{2}(x)<0, \cdots, f_{m}(x)<0 .
$$

Then, either (13) is feasible or there exists some nonzero $\lambda \geq 0$ satisfying

$$
g(\lambda)=\inf _{x}\left\{\lambda_{1} f_{1}(x)+\lambda_{2} f_{2}(x)+\cdots+\lambda_{m} f(x)\right\} \geq 0 .
$$

Exactly one of the above two conditions holds true. The existence of a nonzero $\lambda \geq 0$ satisfying (14) proves the infeasibility of (13). Such a $\lambda$ serves as a certificate of infeasibility. Modern softwares (e.g., SeDuMi [3]) for solving convex optimization problems either generate an optimal solution or a certificate showing infeasibility. In contrast, softwares for nonconvex optimization problems cannot detect infeasibility. They typically fail to converge when the underlying problem is infeasible, either due to data overflow or because the maximum number of iterations is exceeded. 


\section{Linear Conic Optimization Models}

We now review several commonly used convex optimization models in engineering design applications. Consider a primaldual pair of optimization problems

and

$$
\begin{array}{ll}
\text { minimize } & C \bullet X \\
\text { subject to } & \mathcal{A} X=b, \quad X \in \mathcal{K}
\end{array}
$$

$$
\begin{array}{ll}
\text { maximize } & b^{T} y \\
\text { subject to } & \mathcal{A}^{*} y+S=C, \quad S \in \mathcal{K}^{*}
\end{array}
$$

where $\mathcal{A}$ is a linear operator mapping an Euclidean space onto another Euclidean space, $\mathcal{A}^{*}$ denotes the adjoint of $\mathcal{A}, \mathcal{K}$ signifies a pointed, closed convex cone, and $\mathcal{K}^{*}$ is its dual cone. Recall that $C \bullet X$ denotes matrix inner product. The problems (15) and (16) are known as linear conic programming. They include many well-known special cases listed next.

Linear Programming $(L P) \mathcal{K}=\Re_{+}^{n}$ : In this case, the linear conic optimization problem reduces to

$$
\begin{array}{ll}
\operatorname{minimize} & c^{T} x \\
\text { subject to } & A x=b, x \geq 0
\end{array}
$$

and its dual becomes

$$
\begin{array}{ll}
\text { maximize } & b^{T} y \\
\text { subject to } & A^{T} y+s=c, s \geq 0 .
\end{array}
$$

The optimality condition is given by

$$
A x=b, x \geq 0, A^{T} y+s=c, s>0, x^{T} s=0 .
$$

Second-Order Cone Programming $\mathcal{K}=\prod_{i=1}^{n} \operatorname{SOC}\left(n_{i}\right)$ : Let $\tilde{x}=\left(\tilde{x}_{1}, \tilde{x}_{2}, \ldots, \tilde{x}_{k}\right)^{T}$ with $\tilde{x}_{i}=\left(t_{i}, x_{i}\right)^{T} \in$ $\operatorname{SOC}\left(n_{i}\right)$ (namely, $t_{i} \geq\left\|x_{i}\right\|$ ). Similarly, we denote $\tilde{s}=\left(\tilde{s}_{1}, \tilde{s}_{2}, \ldots, \tilde{s}_{k}\right)^{T}$ with $\tilde{s}_{i}=\left(\tau_{i}, s_{i}\right)^{T} \in \operatorname{SOC}\left(n_{i}\right)$. The data vector $\tilde{c}=\left(\tilde{c}_{1}, \tilde{c}_{2}, \ldots, \tilde{c}_{k}\right)^{T}$ with $\tilde{c}_{i} \in \Re^{n_{i}}$, and the data matrix $\tilde{A} \in \Re^{m \times\left(n_{1}+\cdots+n_{k}\right)}$. In this case, the linear conic optimization problem (15) reduces to

$$
\begin{array}{ll}
\text { minimize } & \tilde{c}^{T} \tilde{x} \\
\text { subject to } & \tilde{A} \tilde{x}=b, \tilde{x}_{i} \in \operatorname{SOC}\left(n_{i}\right), \forall i
\end{array}
$$

and its dual becomes

$$
\begin{array}{ll}
\text { maximize } & b^{T} y \\
\text { subject to } & \tilde{A}^{T} y+\tilde{s}=\tilde{c}, \tilde{s}_{i} \in \operatorname{SOC}\left(n_{i}\right), \forall i .
\end{array}
$$

In practice, the second-order cone programming (SOCP) constraints usually take the form of $\left\|A^{\prime} x+b^{\prime}\right\| \leq t^{\prime}$, which can be easily mapped to the form in (19). The optimality condition for (19) is given by

$$
\begin{aligned}
\tilde{A} \tilde{x} & =b, \quad \tilde{x}^{T} \tilde{s}=0 \\
\tilde{A}^{T} y+\tilde{s} & =\tilde{c}, \quad \tilde{x}_{i}, \tilde{s}_{i} \in \operatorname{SOC}\left(n_{i}\right), \forall i .
\end{aligned}
$$

Semidefinite Programming $(S D P) \mathcal{K}=\mathcal{S}_{+}^{n}$ or $\left(\mathcal{H}_{+}^{n}\right)$ : In this case, the linear conic optimization problem reduces to

$$
\begin{array}{ll}
\text { minimize } & C \bullet X \\
\text { subject to } & A_{i} \bullet X=b_{i}, i=1,2, \ldots, m, X \succeq 0
\end{array}
$$

and its dual becomes

$$
\begin{array}{ll}
\text { maximize } & b^{T} y \\
\text { subject to } & \sum_{i=1}^{m} A_{i}^{T} y_{i}+S=C, S \succeq 0 .
\end{array}
$$

In practice, linear matrix inequalities of the form $A_{0}+$ $\sum_{i=1}^{m} y_{i} \succeq 0$ can be easily mapped to the form in (23). The optimality condition is given by

$$
\begin{aligned}
A_{i} \bullet X & =b_{i}, X \succeq 0, \\
\sum_{i=1}^{m} A_{i}^{T} y_{i}+S & =C, S \succeq 0, X \bullet S=0 .
\end{aligned}
$$

\section{Interior-Point Methods for Linear Conic Optimization}

We now discuss numerical algorithms for solving linear conic optimization problems. For ease of exposition, we will focus on the SDP case with $\mathcal{K}=\mathcal{S}_{+}^{n}$. The other cases can be treated similarly (in fact, they are special cases of SDP).

Assume that the feasible regions of the SDP pair (15) and (16) have nonempty interiors. Then, we can define the central path of (15) and (16) as $\{(X(\mu), S(\mu))\}$ satisfying

$$
\begin{aligned}
\mathcal{A}^{*} y(\mu)+S(\mu) & =C \\
\mathcal{A} X(\mu) & =b \\
X(\mu) S(\mu) & =\mu I
\end{aligned}
$$

where $\mu$ is a positive parameter. By driving $\mu \rightarrow 0$ and under mild assumptions, the central path converges to an optimal primal-dual solution pair for (15) and (16). Notice that the central path condition (25) is exactly the necessary and sufficient optimality condition for the following convex problem:

$$
\begin{array}{ll}
\text { minimize } & C \bullet X-\mu \log \operatorname{det}(X) \\
\text { subject to } & \mathcal{A} X=b, X \in \mathcal{S}_{+}^{n} .
\end{array}
$$

In other words, the points on the central path corresponds to the optimal solution of (26) and the associated optimal dual solution. Here, the function $-\log \operatorname{det}(X)$ is called the barrier function for the positive semidefinite matrix cone $\mathcal{S}_{+}^{n}$.

Many interior-point algorithms follow (approximately) the central path to achieve optimality. As a result, the iterates are required to remain in a neighborhood of the central path which can be defined as

$$
\begin{array}{r}
\mathcal{N}(\gamma)=\left\{(X, y, S) \mid \mathcal{A} X=b, \mathcal{A}^{*} y+S=C, X \succeq 0,\right. \\
\left.S \succeq 0,\left\|X^{1 / 2} S X^{1 / 2}-\frac{X \bullet S}{n} I\right\|_{F} \leq \gamma \frac{X \bullet S}{n}\right\} .
\end{array}
$$

With this definition, a generic interior-point path-following algorithm can be stated as follows.

\section{Generic Path-Following Algorithm}

1) Given a strictly feasible primal-dual pair $\left(X^{0}, y^{0}, S^{0}\right) \in \mathcal{N}(\gamma)$ with $0<\gamma<1$. Set $k=0$

2) Let $X=X^{k}, y=y^{k}, S=S^{k}$ and $\mu_{k}=X \bullet S / n$.

3) Compute a search direction $\left(\Delta X^{k}, \Delta y^{k}, \Delta S^{k}\right)$ and the largest step $t_{k}$ such that $\left(X+t^{k} \Delta X^{k}, y+\right.$ $\left.t^{k} \Delta y^{k}, S+t^{k} \Delta S^{k}\right) \in \mathcal{N}(\gamma)$. Set $X^{k+1}=X+t^{k} \Delta X^{k}$, $y^{k+1}=y+t^{k} \Delta y^{k}, S^{k+1}=S+t^{k} \Delta S^{k}$.

4) Set $k=k+1$ and return to 2 ) until convergence. 


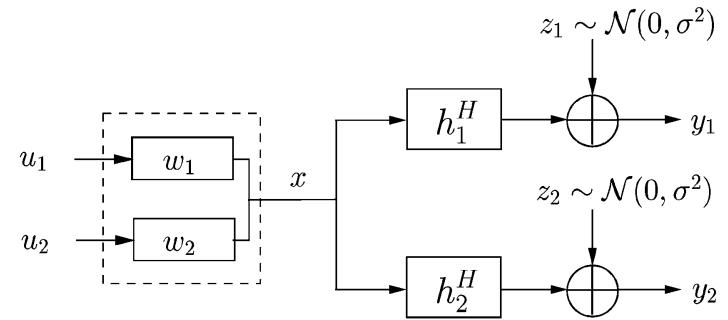

$\min \sum_{i}\left\|w_{i}\right\|^{2}$

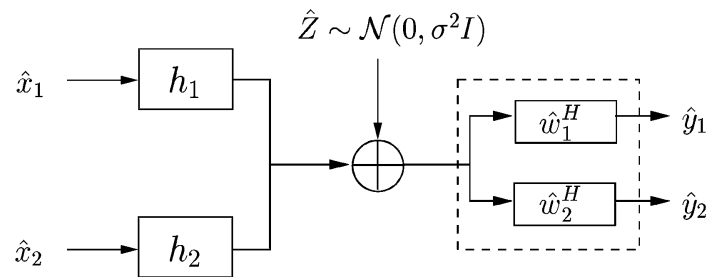

$\min \sum_{i} \mathbb{E}\left[\hat{x}_{i}^{2}\right]$

Fig. 1. Uplink-downlink beamforming duality can be interpreted as a Lagrangian duality in convex optimization.

There are many choices for the search direction $(\Delta X, \Delta y, \Delta S)$. For example, we can take it as the solution of the following linear system of equations:

$$
\begin{aligned}
\mathcal{A}^{*} \Delta y+\Delta S & =C-S-\mathcal{A}^{*} y \\
\mathcal{A} \Delta X & =b \\
\mathcal{H}_{P}(\Delta X S+X \Delta S) & =\mu I-\mathcal{H}_{P}(X S)
\end{aligned}
$$

where $P$ is a nonsingular matrix and

$$
\mathcal{H}_{P}(U)=\frac{1}{2}\left(P U P^{-1}+\left(P U P^{-1}\right)^{T}\right)
$$

Different choices of $P$ lead to different search directions. For example, $P=I$ corresponds to the so-called AlizadehHaeberly-Overton (AHO) direction [2].

The standard analysis of path-following interior-point methods shows that a total of $O\left(\sqrt{n} \log \mu_{0} / \epsilon\right)$ main iterations are required to reduce the duality gap $X \bullet S$ to less than $\epsilon$. Each main iteration involves solving the linear system of (28) whose size depends on the underlying cone $\mathcal{K}$. If $\mathcal{K}=\Re_{+}^{n}$ (linear programming), the linear system is of size $O(n)$, implying each main iteration has an arithmetic complexity of $O\left(n^{3}\right)$. In the case where $\mathcal{K}=\prod_{i=1}^{n} \operatorname{SOC}\left(n_{i}\right)$ (SOCP), the linear system (28) will have size $O\left(\sum_{i} n_{i}\right)$, so the complexity of solving (28) is $O\left(\left(\sum_{i} n_{i}\right)^{3}\right)$. For the SDP case where $\mathcal{K}=\mathcal{S}_{+}^{n}$, the size of the linear system (28) is $O\left(n^{2}\right)$, so the amount of work required to solve (28) is $O\left(n^{6}\right)$. Combining the estimates of the number of main iterations with the complexity estimate per each iteration yields the overall complexity of interior-point methods. In general, the computational effort required to solve SDP is more than that of SOCP, which in turn is more than that of LP. However, the expressive powers of these optimization models rank in the reverse order.

The brief overview here is by no means exhaustive. Several important classes of convex problems, such as geometric programming [4], have not been included. The readers are referred to many excellent texts (e.g., [5]) in this area.

\section{CONIC Programming For Multiuser BeAmForming}

The rest of this paper treats several applications of convex optimization in communications and signal processing to illustrate the concepts covered so far. Communication problems often involve the optimization of some design objective, such as transmit power or the detection probability of error, subject to resource constraints, such as bandwidth and power. Traditional optimization methods in communications focus on analytic or adaptive solutions to the problem - as in minimum mean-squared error (MMSE) equalizer design. The MMSE problem is essentially a convex quadratic program $(\mathrm{QP})$. This section focuses on the application of modern techniques such as linear conic programming.

\section{A. Downlink Beamforming Problem as SDP and SOCP}

A key step in the application of convex optimization techniques in engineering problems is the formulation of the problem in a convex form. This can be nontrivial. This section illustrates this step using an example from wireless communications.

Consider a transmitter beamforming problem for a wireless downlink channel in which the base station is equipped with multiple antennas and each remote user is equipped with a single antenna, as shown in Fig. 1. The channel is modeled as

$$
y_{i}=h_{i}^{H} x+z_{i}, \quad i=1, \ldots, K
$$

where $x \in \mathbb{C}^{n}$ represents the transmit signal, $h_{i}^{H} \in \mathbb{C}^{n}$ are channel vectors assumed to be known to all the transmitter and the receivers, and $z_{i}$ 's are the independent identically distributed (i.i.d.) additive complex Gaussian noises with variance $\sigma^{2} / 2$ on each of its real and imaginary components. For now, we restrict our attention to the single-receive-antenna case in which $y_{i}$ 's are complex scalars.

In a beamforming design, the transmit signal is of the form $x=\sum_{i=1}^{K} \nu_{i} w_{i}$, where $\nu_{i}$ is a complex scalar denoting the information signal for user $i$, and $w_{i} \in \mathbb{C}^{n}$ is the beamforming vector for user $i$. Without loss of generality, let $\mathbb{E}\left|\nu_{i}\right|^{2}=1$. The received signals are $y_{i}=h_{i}^{H}\left(\sum_{j=1}^{K} \nu_{j} w_{j}\right)+z_{i}, i=1, \cdots, K$. The signal-to-interference-and-noise ratio (SINR) for each user is expressed as

$$
\operatorname{SINR}_{i}=\frac{\left|h_{i}^{H} w_{i}\right|^{2}}{\sum_{j \neq i}\left|h_{i}^{H} w_{j}\right|^{2}+\sigma^{2}} .
$$


An important class of optimal downlink beamforming problem involves finding a set of $w_{i}$ 's that minimizes the total transmit power, while satisfying a given set of SINR constraints $\gamma_{i}$

$$
\begin{aligned}
\operatorname{minimize} & \sum_{j=1}^{K}\left\|w_{j}\right\|^{2} \\
\text { subject to } & \frac{\left|h_{i}^{H} w_{i}\right|^{2}}{\sum_{j \neq i}\left|h_{i}^{H} w_{j}\right|^{2}+\sigma^{2}} \geq \gamma_{i}, \quad \forall i .
\end{aligned}
$$

For simplicity, we make the assumption that the set of $\gamma_{i}$ is feasible.

As stated in (32), the SINR constraint is not convex. However, this seemingly nonconvex problem has many of the features of convex problems. For example, as shown in [6]-[9], this downlink problem can be solved in an iterative fashion via a so-called uplink-downlink duality. Further, it turns out that the above problem can be relaxed [10] or transformed [11] into a convex optimization problem. This section gives an overview of the approaches in [10] and [11] to illustrate the application of convex optimization. In the next section, we take advantage of the convex formulation and illustrate an engineering interpretation of Lagrangian duality in this setting.

One approach to solving the beamforming problem is based on a reformulation of (31) in terms of new matrix variables $B_{i}=$ $w_{i} w_{i}^{H}$ [10]. Clearly, $B_{i}$ is a positive semidefinite matrix. Define $H_{i}=h_{i} h_{i}^{H}$. The optimization problem (31), in terms of $B_{i}$, then becomes the following SDP

$$
\begin{array}{ll}
\text { minimize } & \sum_{i=1}^{K} \operatorname{tr}\left(B_{i}\right) \\
\text { subject to } & \operatorname{tr}\left(H_{i} B_{i}\right)-\gamma_{i} \sum_{j \neq i} \operatorname{tr}\left(H_{i} B_{j}\right) \geq \gamma_{i} \sigma^{2} \\
& B_{i} \succeq 0, \quad B_{i} \text { is complex Hermitian. }
\end{array}
$$

However, the original problem (31) requires the optimal solution $w_{i} w_{i}^{H}$ to be rank-1, which is not guaranteed a priori in the above formulation. In effect, the above formulation relaxes the rank-1 constraint. For this reason, (33) is referred to as a SDP relaxation.

Surprisingly, as shown by Bengtsson and Ottersten [10], the above SDP relaxation is guaranteed to have at least one optimal solution which is rank one. This unexpected property lends tremendous power of convex optimization to this apparently nonconvex problem. In Section IV-B, we will return to SDP relaxation and consider a closely related multicast problem for which this property does not hold.

The fundamental reason that the SDP relaxation is optimal for the above problem is that this problem can be reformulated as a convex problem. This is shown in [11] using SOCP. Observe that an arbitrary phase rotation can be added to the beamforming vectors without affecting the SINR. Thus, $h_{k}^{H} w_{k}$ can be chosen to be real without the loss of generality, Now, let $W=\left[w_{1}, \ldots, w_{K}\right]$. The SINR constraints become

$$
\left(1+\frac{1}{\gamma_{i}}\right)\left|h_{i}^{H} w_{i}\right|^{2} \geq\left\|{ }_{\sigma}^{h_{i}^{H} W}\right\|^{2}, \forall i
$$

Because $h_{k}^{H} w_{k}$ can be assumed to be real, we may take the square root of the above equation. The constraint becomes a second-order cone constraint, which is convex. The optimization problem now becomes

$$
\begin{array}{ll}
\text { minimize } & \tau \\
\text { subject to } & \sqrt{1+\frac{1}{\gamma_{i}}} h_{i}^{H} w_{i} \geq\left\|\begin{array}{c}
h_{i}^{H} W \\
\sigma
\end{array}\right\|, \forall i \\
& \sum_{j=1}^{K}\left\|w_{j}\right\| \leq \tau
\end{array}
$$

which is in the form of a second-order cone program (19).

\section{B. Uplink-Downlink Duality via Lagrangian Duality}

In engineering design, one is often interested in not only the numerical solution to the problem, but also the structure of the optimal solution. When a problem is formulated as a convex optimization problem, exploring its dual often reveals such structures. In this section, we illustrate this point by showing that the Lagrangian dual of the SOCP problem [11] has an engineering interpretation, which is known as uplink-downlink [6], [7], [9], [12], [13].

Several different versions of uplink-downlink duality have been developed in the literature. In the beamforming context, uplink-downlink duality refers to the fact that the minimum power needed to achieve a certain set of SINR targets in a downlink multiple-input-multiple-output (MIMO) channel is the same as the minimum power needed to achieve the same set of SINR targets in the uplink channel, where the uplink channel is derived by reversing the input and the output of the downlink [6]-[9], [14]. A very similar uplink-downlink duality can be derived from an information theory point of view. As shown in [12], [13], [15], and [16], the capacity region of a downlink broadcast channel is identical to the capacity region of an uplink channel with the same sum power constraint. These two duality results can be unified via Lagrangian duality in convex optimization, first shown in [17]. The following outlines the main steps.

We begin the development by writing down the Lagrangian of the downlink beamforming problem $(31)^{2}$ :

$$
\begin{aligned}
L\left(w_{i}, \lambda_{i}\right)= & \sum_{j=1}^{K} w_{j}^{H} w_{j}-\sum_{i=1}^{K} \lambda_{i} \\
& \times\left\{\frac{1}{\gamma_{i}}\left|h_{i}^{H} w_{i}\right|^{2}-\sum_{j \neq i}\left|h_{i}^{H} w_{j}\right|^{2}-\sigma^{2}\right\} \\
= & \sum_{i=1}^{K} \lambda_{i} \sigma^{2}+\sum_{i=1}^{K} w_{i}^{H} \\
& \left\{I+\sum_{j \neq i} \lambda_{j} h_{j} h_{j}^{H}-\frac{\lambda_{i}}{\gamma_{i}} h_{i} h_{i}^{H}\right\} w_{i} .
\end{aligned}
$$

${ }^{2}$ Technically, we should take the dual of (35), as an optimization problem may, in genera, 1 be written in different forms, each of which may lead to a different dual. In this case, the duals of (31) and (35) turn out to be the same. 
The dual objective is

$$
g\left(\lambda_{i}\right)=\min _{w_{i}} L\left(w_{i}, \lambda_{i}\right)
$$

It is easy to see that if $I+\sum_{j \neq i} \lambda_{j} h_{j} h_{j}^{H}-\left(\lambda_{i} / \gamma_{i}\right) h_{i} h_{i}^{H}$ is not a positive semidefinite matrix, then there exists a set of $w_{i}$ which would make $g\left(\lambda_{i}\right)=-\infty$. As $\lambda_{i}$ should be chosen to maximize $g\left(\lambda_{i}\right)$, the second term in (36) leads to a positive semidefinite constraint in the dual problem. In particular, the Lagrangian dual problem is

$$
\begin{array}{ll}
\operatorname{maximize} & \sum_{i=1}^{K} \lambda_{i} \sigma^{2} \\
\text { subject to } & \sum_{j=1}^{K} \lambda_{j} h_{j} h_{j}^{H}+I \succeq\left(1+\frac{1}{\gamma_{i}}\right) \lambda_{i} h_{i} h_{i}^{H} .
\end{array}
$$

Interestingly, the above dual problem can be shown to correspond to an uplink problem with $\lambda_{i}$ as the (scaled) uplink power, $h_{j}$ as the uplink channel, and $\gamma_{i}$ as the SINR constraint.

The uplink channel is depicted in Fig. 1. The sum power minimization problem for the uplink channel can be formulated as

$$
\begin{aligned}
\operatorname{minimize} & \sum_{i=1}^{K} \rho_{i} \\
\text { subject to } & \frac{\rho_{i}\left|\hat{w}_{i}^{H} h_{i}\right|^{2}}{\sum_{j \neq i} \rho_{j}\left|\hat{w}_{i}^{H} h_{j}\right|^{2}+\sigma^{2} \hat{w}_{i}^{H} \hat{w}_{i}} \geq \gamma_{i}
\end{aligned}
$$

where the optimization is over the uplink power $\rho_{i}$ and the receiver beamforming vectors $\hat{w}_{i}$. Clearly, the optimal $\hat{w}_{i}$ is just the MMSE filter $\hat{w}_{i}=\left(\sum_{j=1}^{K} \rho_{i} h_{j} h_{j}^{H}+\sigma^{2} I\right)^{-1} h_{i}$. Substituting the MMSE filter into the constraints of (39) and after a matrix manipulation, it is possible to show that the uplink problem is equivalent to

$$
\begin{array}{ll}
\text { minimize } & \sum_{i=1}^{K} \rho_{i} \\
\text { subject to } & \sum_{j=1}^{K} \rho_{j} h_{j} h_{j}^{H}+\sigma^{2} I \preceq\left(1+\frac{1}{\gamma_{i}}\right) \rho_{i} h_{i} h_{i}^{H} .
\end{array}
$$

Identifying $\rho_{i}=\lambda_{i} \sigma^{2}$, we see that (40) is identical to (38), except that the maximization and the minimization are reversed and the SINR constraints are also reversed. In a downlink beamforming problem, the SINR constraints are always met with equality. Thus, the maximization problem (38) and the minimization problem (40), in fact, give the same solution. Finally, because strong duality holds for this problem, the primal and the dual problem must have the same solution. It is interesting to note that the dual variables of the downlink problem have the interpretation of being the uplink power scaled by the noise variance.

As mentioned earlier, this uplink-downlink duality has been derived earlier in the literature [6]-[9], [14]. This section reinterprets this duality as an example of Lagrangian duality to illustrate the use of convex optimization. The duality can be further enhanced if we also take different base-station antenna constraints into consideration. This is explored in the recent work [17]. Duality is useful because the uplink problem is easier to solve. For example, an iterative optimization of $\rho_{i}$ and $w_{i}$ leads to the optimal solution for the downlink [6], [7], [9]. Algorithms based on convex optimization have been considered in [11] and [17].

\section{Capacity Region Duality}

Instead of considering a power minimization problem subject to SINR constraints, in many applications, it is useful to consider the reverse problem of maximizing a rate region, subject to a power constraint. Under many practical coding and modulation schemes, the SINR can be directly related to the achievable rate using an SNR-gap approximation: $R=\log (1+\operatorname{SINR} / \Gamma)$, where $\Gamma$ is the gap to capacity, which is always greater than 1 . The SINR duality stated in the previous section directly leads to a rate-region duality as well. The argument follows from the fact that since the minimum transmit power for each fixed set of SINRs is the same for both uplink and downlink, the set of achievable SINR regions under a fixed power must also be the same in both uplink and downlink. Therefore, the rate regions must be the same.

This argument can be further generalized to a downlink channel with multiple receive antennas for each user, and it directly leads to the information theoretical duality of the multiple-access channel and the broadcast channel [12], [13] by letting $\Gamma=1$ and by using interference subtraction techniques. Interference can be directly subtracted in the multiple-access channel at the receiver, and can be presubtracted at the transmitter in the broadcast channel. The previous proof of this capacity region uplink-downlink duality relies on an intricate transmit covariance matrix transformation. The Lagrangian approach shows an alternative way of deriving the same thing.

For completeness, the most general capacity region duality [12], [13] is stated as follows. Consider a MIMO downlink channel where each user is equipped with multiple antennas

$$
y_{i}=H_{i} x+z_{i}, \quad i=1, \ldots, K
$$

where $H_{i}$ 's are now matrices and $y_{i}$ 's complex vectors. The capacity region of the downlink channel can be found by solving the following weighted rate-sum optimization problem:

$$
\begin{array}{ll}
\text { maximize } & \sum_{k=1}^{K} \mu_{k} \log \frac{\left|H_{k}\left(\sum_{i=1}^{k} S_{i}\right) H_{k}^{H}+I\right|}{\left|H_{k}\left(\sum_{i=1}^{k-1} S_{i}\right) H_{k}^{H}+I\right|} \\
\text { subject to } & \sum_{i=1}^{K} \operatorname{tr}\left(S_{i}\right) \leq \rho_{T}, \quad S_{i} \succeq 0
\end{array}
$$

where $\rho_{T}$ is the total power constraint. The optimal weighted rate-sum turns out to be exactly the same as the optimal weighted rate-sum of a dual multiple-access channel under the same total power constraint

$$
\hat{y}=\sum_{i=1}^{K} H_{i}^{H} \hat{x}_{i}+\hat{z} .
$$


The capacity region for the multiple-access channel may be expressed as

$$
\begin{array}{ll}
\text { maximize } & \sum_{k=1}^{K} \mu_{k} \log \frac{\left|\sum_{i=1}^{k} H_{i}^{H} \hat{S}_{i} H_{i}+I\right|}{\left|\sum_{i=1}^{k-1} H_{i}^{H} \hat{S}_{i} H_{i}+I\right|} \\
\text { subject to } & \sum_{i=1}^{K} \operatorname{tr}\left(\hat{S}_{i}\right) \leq \rho_{T}, \quad \hat{S}_{i} \succeq 0 .
\end{array}
$$

Here, $S_{i}$ and $\hat{S}_{i}$ are the transmit covariance matrices for user $i$ in the downlink and in the uplink, respectively; all noise vectors are assumed to be i.i.d. with unit variance; $\mu_{1} \geq \mu_{2} \geq \ldots \geq \mu_{K} \geq$ 0 are weights characterizing different boundary points of the capacity region. Again, this duality is useful because the multiple-access channel problem (44) is convex, while the broadcast channel problem (42) is not. This fact has been exploited in [18] and [19] for efficient computation of the sum capacity. The authors are not yet aware of a direct Lagrangian duality relation between (42) and (44), except for the sum capacity case for which a Lagrangian interpretation has been given in [20]. It would be interesting to see whether it is possible to find a way to do so for the entire capacity region.

\section{Open Problems}

Optimization is expected to play an increasingly important role in multiuser MIMO system design. As the previous sections illustrate, for both the uplink and downlink scenarios, the capacity maximization problem (subject to power constraint) and the power minimization problem (subject to SINR constraints) can both be formulated as a convex optimization problem. Thus, both problems can be considered as solved. However, the SINRconstrained power minimization solution is applicable only to the single-remote-antenna case. When the remote users have multiple antennas as well, it can be shown that a duality still exists, but an optimal solution is still lacking.

An important but difficult problem in this area is that of the interference channel, where multiple transmitters and multiple receivers interfere with each other in a shared medium. Even for the single-antenna case, the rate maximization problem is not yet amendable to a convex formulation. The fundamental problem is that the achievable rate expression

$$
R=\log \left(1+\frac{\rho_{i} G_{i i}}{\rho_{j} G_{j i}+\sigma^{2}}\right)
$$

where $\left[G_{i j}\right]$ is the channel coupling matrix, is not a concave function of $\rho_{j}$. (Nevertheless, a network duality result is still available [21].) Recent progress has been made in this area for both wireline [22]-[24] and wireless applications [25]. Further progress is still needed.

\section{SDP RELAXATIONS FOR NONCONVEX PROBLEMS}

Convex optimization can also play an important role in inherently nonconvex problems. In this case, the method of relaxation can produce excellent results. This section gives two such examples and discusses open issues and future directions in this area.

\section{A. Multiuser Detection}

Consider a detection and estimation problem in the receiver processing of a MIMO channel

$$
y=\sqrt{\rho / n} H s+z
$$

where $\rho$ is the (normalized) average SNR at each receive antenna, $H \in \mathbb{C}^{n \times m}$ denotes the (known) channel matrix, $y \in$ $\mathbb{C}^{n}$ is the received channel output, $s$ is the transmitted information binary symbol vector from the signal constellation set $\{-1,1\}^{m}$, and $z$ denotes the additive white Gaussian channel noise with unit variance. The capacity of this MIMO channel is known to be proportional to the number of transmit antennas. To reap the benefits brought by the MIMO channel, one must develop an efficient detection algorithm for such a system.

Central to the MIMO maximum-likelihood (ML) channel detection is the following constrained optimization problem:

$$
f_{\mathrm{ML}}:=\min _{x \in\{-1,1\}^{m}}\|y-\sqrt{\rho / n} H s\|^{2}
$$

where $s$ is the unknown (to be determined) transmitted binary information vector.

A popular method to solve (47) is with the sphere decoder [26]. This method, while enjoying a good practical complexity/ performance tradeoff for small system size and high SNR, is known to have exponential average-case complexity for each fixed SNR value [27]. Below, we describe a semidefinite programming relaxation method, which provides an effective polynomial-time approach for this problem.

For simplicity, consider the case $m=n$ and $H, s$ and $z$ are real; the extension to the general case is relatively simple. First, we rewrite the log-likelihood function as

$$
\|y-\sqrt{\rho / n} H s\|^{2}=\operatorname{tr}\left(Q x x^{T}\right)
$$

where matrix $Q \in \Re^{(n+1) \times(n+1)}$ and vector $x \in \Re^{n+1}$ are defined as

$$
Q=\left[\begin{array}{cc}
(\rho / n) H^{T} H & -\sqrt{\rho / n} H^{T} y \\
-\sqrt{\rho / n} y^{T} H & \|y\|^{2}
\end{array}\right], x=\left[\begin{array}{l}
s \\
1
\end{array}\right]
$$

Let $X=x x^{T}$ and notice that $X \succeq 0, X_{i i}=1$ and $\operatorname{rank}(X)=1$ if and only if $X=x x^{T}$ for some $x$ with $x_{i}= \pm 1$. By rewriting (47) in terms of $X$ and relaxing the rank-1 constraint, we arrive at a SDP relaxation for (47)

$$
\begin{aligned}
f_{\mathrm{SDP}}:= & \min \quad \operatorname{tr}(Q X), \\
& \text { s.t. } \quad X \succeq 0, X_{i, i}=1, \forall i .
\end{aligned}
$$

Once the optimal solution $X_{\text {opt }}$ of (49) is computed, we use the following randomized procedure to generate a feasible rank-1 solution $\hat{x}_{\mathrm{SDR}}$.

1) Compute the largest eigenvalue of $X_{o p t}$ and the associated eigenvector $v=\left(v_{1}, v_{2}, \ldots, v_{n+1}\right)^{T}$.

2) Generate $L$ i.i.d. binary vector samples $\bar{x}_{\ell}, \ell=1, \ldots, L$, whose $i$ th entry $(i=1, \ldots, n+1)$ follows the distribution:

$$
\begin{aligned}
& \operatorname{Pr}\left\{x_{i}=+1\right\}=\left(1+v_{i}\right) / 2 \\
& \operatorname{Pr}\left\{x_{i}=-1\right\}=\left(1-v_{i}\right) / 2 .
\end{aligned}
$$




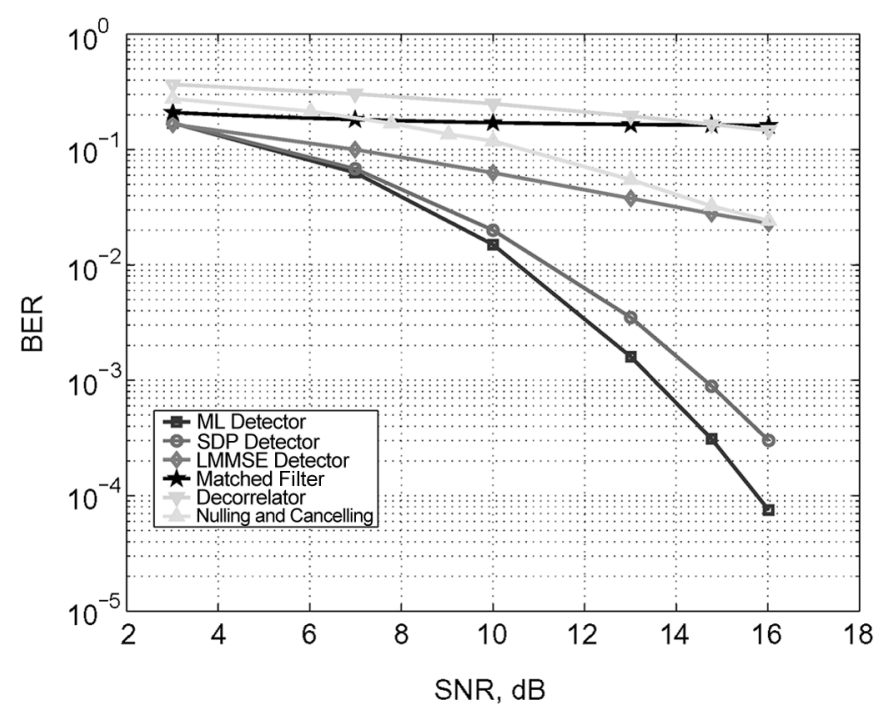

Fig. 2. BER as a function of SNR for different detectors. Simulation parameters: BPSK modulation $n=10$.

3) Pick $\hat{x}:=\arg \min _{\ell} \bar{x}_{\ell}^{T} Q \bar{x}_{\ell}$ and assign $f_{\mathrm{SDR}}:=\hat{x}^{T} Q \hat{x}$ and set $\hat{x}_{\mathrm{SDR}}$ (the quasi-ML estimate) to be the first $n$-entries of $\hat{x}$ multiplied by its last entry (to correct the sign).

Due to the diagonal structure of the constraints, the SDP (49) can be efficiently solved with a worst-case complexity of $O\left(n^{3.5}\right)$ (rather than $O\left(n^{6.5}\right)$ for a general SDP). The complexity of the randomization procedure is negligible. (Theoretically, $L$ should be chosen as a polynomial of $n$, but practically is usually set to 10-30.) So the overall complexity of SDP detector is $O\left(n^{3.5}\right)$.

Compared with the existing polynomial-time linear suboptimal MIMO detectors, the above SDP detector performs surprisingly well, offering an excellent performance-complexity tradeoff in practical SNR ranges; see Fig. 2. Moreover, compared with the sphere decoder which has exponential averagecase complexity, the polynomial-time SDP detector runs faster when the problem size becomes large and SNR is low (e.g., $\rho=10 \mathrm{~dB}$ and $n \geq 45$ ).

An interesting research direction is to investigate the averagecase performance of SDP relaxation for large systems. In particular, it is interesting to see if the gap in bit-error rate (BER) between the ML curve and the SDP curve in Fig. 2 remains bounded for large $n$. An affirmative answer to this question could lead to the first polynomial-time quasi-ML detector which is guaranteed to offer a bounded SNR loss for large systems. Some encouraging results in this direction can be found in [28], where a probabilistic analysis of the SDP relaxation method has been given for a standard Rayleigh flat-fading MIMO channel model under additive Gaussian noise model. In particular, it has been shown that for any fixed $\rho>0$, the average ratio $f_{\mathrm{SDR}} / f_{\mathrm{ML}}$ remains bounded by a positive constant (depending on $\rho$ ) for all $n>0$.

\section{B. Multicast Beamforming}

We now return to the multiuser transmit beamforming problem of Section III-A, but consider a broadcast application [29] in which a transmitter utilizes an array of $n$ transmitting antennas to simultaneously broadcast common information to $m$ radio receivers, with each receiver $i \in\{1, \ldots, m\}$ equipped with $\left|I_{i}\right|$ receiving antennas. Let $h_{\ell}, \ell \in I_{i}$, denote the $n \times 1$ complex channel vector modeling propagation loss and phase shift from the transmitting antennas to the $\ell$ th receiving antenna of receiver $i$. Assume that the transmitter uses a single beamforming vector $w$ to transmit common information to all receivers and each receiver performs optimal matched filtering and maximum-ratio combining. Then, the constraint

$$
\sum_{\ell \in I_{i}}\left|h_{\ell}^{H} w\right|^{2} \geq 1
$$

models the requirement that the total received signal power at receiver $i$ must be above a given threshold (normalized to 1). This is equivalent to the SNR constraint considered in Section III-A (but without interference). To minimize the total transmit power subject to individual SNR requirements (one per each receiver), we are led to the following nonconvex QP

$$
\begin{aligned}
v_{\mathrm{qp}}:=\min & \|w\|^{2} \\
\text { s.t. } & w^{H} H_{i} w \geq 1, \quad i=1,2, \ldots, m
\end{aligned}
$$

where $H_{i}:=\sum_{\ell \in I_{i}} h_{\ell} h_{\ell}^{H}$. Unlike the independent-information problem considered in Section III-A, the above problem has no known convex reformulation.

Let $B=w w^{H}$. The SDP relaxation of (51) is

$$
\begin{aligned}
v_{\text {sdp }}:=\min & \operatorname{tr}(B) \\
\text { s.t. } & \operatorname{tr}\left(H_{i} B\right) \geq 1, \quad i=1, \ldots, m, \\
& B \succeq 0, \quad B \text { is complex Hermitian. }
\end{aligned}
$$

Since we assume $H_{i} \neq 0$ for all $i$, it is easily checked that (52) has an optimal solution, which we denote by $B^{*}$.

Upon obtaining an optimal solution $B^{*}$ of (52), we construct a feasible solution of (51) using the following randomized procedure.

1) Generate a random vector $\xi \in \mathbb{C}^{n}$ from the complexvalued normal distribution $N_{c}\left(0, B^{*}\right)$.

2) Let $w^{*}(\xi)=\xi / \min _{1 \leq i \leq m} \sqrt{\xi^{H} H_{i} \xi}$.

Experimentally, this relaxation approach works very well. For example, in experiments with measured digital subscriber line channel data [29], $v_{\mathrm{qp}} / v_{\mathrm{sdp}}=1$ in over $50 \%$ of instances. We also observed that semidefinite relaxation can almost double the minimum received signal power relative to no precoding.

Recent theoretical analysis in [30] shows that the worst-case performance ratio $v_{\mathrm{qp}} / v_{\mathrm{sdp}}$ can be bounded as follows:

$$
\frac{m}{2 \pi^{2}(2+\pi / 2)^{2}} \leq \frac{v_{\mathrm{qp}}}{v_{\mathrm{sdp}}} \leq 8 \mathrm{~m} .
$$

In other words, the worst-case performance ratio deteriorates linearly with the number of users in the system. For the problem of transmit beamforming for broadcasting with single receiving antenna per subscriber node, simulations have confirmed the above worst-case analysis of the performance ratio $v_{\mathrm{qp}} / v_{\mathrm{sdp}}$; see Fig. 3. Interestingly, when matrices $H_{i}$ and the vector $z$ are 


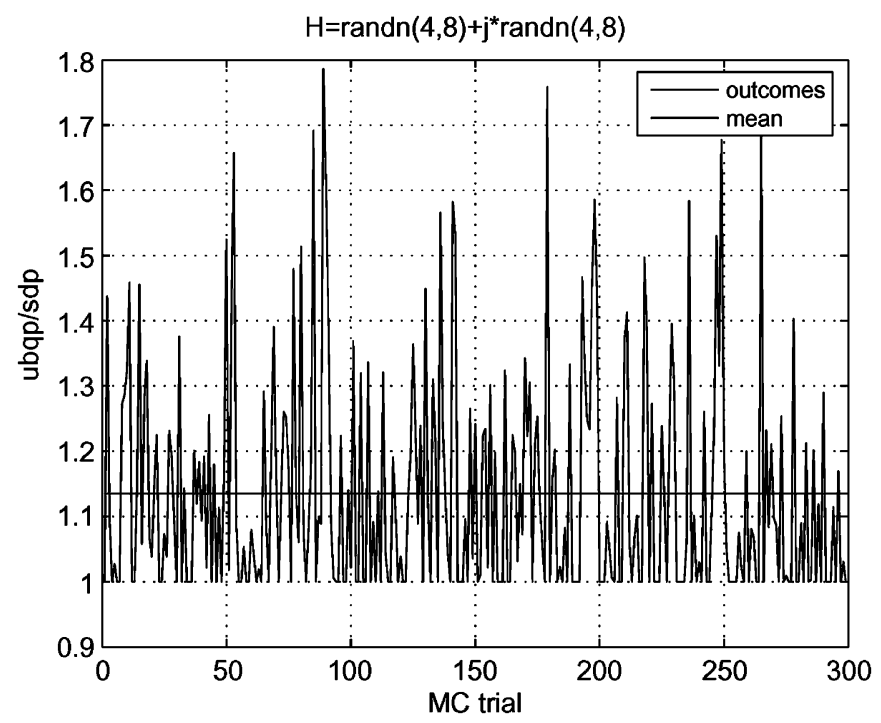

Fig. 3. Upper bound on $v_{\mathrm{qp}} / v_{\mathrm{sdp}}$ for $m=8, n=4$, over 300 realizations of complex Gaussian i.i.d. channel vector entries.

required to be real-valued, then the above approximation ratio becomes quadratic in $m$ [30].

\section{Future Research}

An interesting future direction is to generalize the above formulation to a situation of $1 \leq G \leq M$ multicast groups, $\left\{\mathcal{G}_{1}, \ldots, \mathcal{G}_{G}\right\}$, where $\mathcal{G}_{k}$ is the index set for receivers participating in multicast group $k$, and $k \in\{1, \ldots, G\}$. Common information is transmitted to members within each group; independent information is transmitted to different groups. Assume that $\mathcal{G}_{k} \cap \mathcal{G}_{\ell}=\emptyset, \ell \neq k, \cup_{k} \mathcal{G}_{k}=\{1, \ldots, M\}$. Denote $G_{k}:=\left|\mathcal{G}_{k}\right|, \sum_{k=1}^{G} G_{k}=M$.

Let $w_{k} \in \mathbb{C}^{n}$ denote the beamforming weight vector applied to the $n$ transmitting antenna elements to transmit multicast stream $k$. The signal transmitted by the antenna array is equal to $\sum_{k=1}^{G} w_{k}^{H} s_{k}$, where $s_{k}$ is the information-bearing signal directed to multicast group $k$. This setup includes the case of broadcasting $G=1$ (51) and the case of individual transmissions $G=M(31)$ as special cases. If $s_{k}$ 's are i.i.d. with zero-mean and unit variance, then the total transmit power is equal to $\sum_{k=1}^{G}\left\|w_{k}\right\|^{2}$. The joint design of transmit beamformers subject to received SINR constraints can be posed as follows:

$$
\begin{aligned}
& \min _{\left\{w_{k} \in \mathbb{C}^{n}\right\}_{k=1}^{G}} \sum_{k=1}^{G}\left\|w_{k}\right\|^{2} \\
& \text { s.t. } \frac{\left|w_{k}^{H} h_{\ell}\right|^{2}}{\sum_{i \neq k}\left|w_{i}^{H} h_{\ell}\right|^{2}+\sigma_{\ell}^{2}} \geq c_{\ell}, \\
& \forall \ell \in \mathcal{G}_{k}, \forall k \in\{1, \ldots, G\} .
\end{aligned}
$$

It can be seen that this problem is exactly the kind of nonconvex QPs considered in (51) except that here $H_{i}$ is no longer positive semidefinite. Simulation results show that the corresponding SDP relaxation (52) still provides an excellent solution for this multigroup multicast beamforming problem.

The beamforming problem (53) is NP-hard in general (even the single group case (51) is NP-hard); see [29]. However, there are several special cases of (53) which are polynomial-time solvable. For example, as discussed in Section III-A, the SDP relaxation of (53) is exact when $\left|\mathcal{G}_{k}\right|=1$ for all $k$ (i.e., each group has exactly one user); so this is a polynomial-time solvable case. Another efficiently solvable case is when the channel vectors $h_{\ell}$ 's have the Vandermonde structure $\left[1 e^{j \theta_{\ell}} e^{j 2 \theta_{\ell}} \cdots e^{j(n-1) \theta_{\ell}}\right]^{T}$, as in the case of a uniform linear transmitting antenna array. In this case, the SDP relaxation of (53) is again tight. It would be interesting to analyze the worst-case performance of the SDP relaxation algorithm of the general homogeneous ${ }^{3}$ nonconcave (and nonconvex) QP.

\section{Robust OptiMIZATION}

Robust optimization models in mathematical programming have received much attention recently; see, e.g., [31]-[33]. In this section, we briefly review some of these models and their extensions.

Consider a convex optimization of the form

$$
\begin{array}{ll}
\operatorname{minimize} & f_{0}(x) \\
\text { subject to } & f_{i}(x) \leq 0, \quad i=1,2, \ldots, m
\end{array}
$$

where each $f_{i}$ is convex. In many engineering design applications, the data defining the constraint and the objective functions may be inexact, corrupted by noise, or may fluctuate with time around a nominal value. In such cases, the traditional optimization approach simply solves (54) by using the nominal value of the data. However, an optimal solution for the nominal formulation (54) may yield poor performance or become infeasible when each $f_{i}$ is perturbed in the actual design. A more appropriate design approach is to seek a high-quality solution which can remain feasible and deliver high-quality performance in all possible realizations of unknown perturbations. This principle was formulated rigorously in [31]-[33]. Specifically, we consider a family of perturbed functions parameterized by $\delta: f_{i}(x ; \delta)$, with $\delta$ taken from an uncertainty set $\Delta$. Then, a robustly feasible solution $x$ is the one that satisfies

$$
f_{i}(x ; \delta) \leq 0, \quad \forall \delta \in \Delta \text { or equivalently } \max _{\delta \in \Delta} f_{i}(x ; \delta) \leq 0 .
$$

Thus, a robustly feasible solution $x$ is, in a sense, strongly feasible, since it is required to satisfy all slightly perturbed version of the nominal constraint. The robust optimal solution can now be defined as a robust feasible solution which minimizes the worst-case objective value $\max _{\delta \in \Delta} f_{0}(x ; \delta)$. This gives rise to the following formulation:

$$
\begin{array}{ll}
\operatorname{minimize} & \max _{\delta \in \Delta} f_{0}(x ; \delta) \\
\text { subject to } & f_{i}(x ; \delta) \leq 0, \forall \delta \in \Delta, i=1,2, \ldots, m .
\end{array}
$$

Let us assume the perturbation vector $\delta$ enters the objective and the constraint functions $f_{i}$ in such a way that preserves convexity, i.e., each $f_{i}(x ; \delta)$ remains a convex function for each $\delta \in \Delta$. In this case, the robust counterpart (55) of the original (nominal case) convex problem (54) remains convex since its constraints are convex (for each $i$ and $\delta$ ) and the objective function $\max _{\delta \in \Delta} f_{0}(x ; \delta)$ is also convex.

\footnotetext{
${ }^{3} \mathrm{~A} \mathrm{QP}$ is homogeneous if it does not have linear terms. A homogeneous QP always has an SDP relaxation.
} 
Much of the research in robust optimization focuses on finding a finite representation of the feasible region of (55) which is defined in terms of infinitely many constraints (one for each $\delta \in \Delta$ ). Assume that the uncertainty parameter $\delta$ can be partitioned as $\delta=\left(\delta_{0}, \delta_{1}, \delta_{2} \ldots, \delta_{m}\right)^{T}$ and that the uncertainty set has a Cartesian product structure $\Delta=\Delta_{0} \times \Delta_{1} \times \cdots \times \Delta_{m}$, with $\delta_{i} \in \Delta_{i}$. Moreover, assume that $\delta$ enters $f_{i}(x ; \delta)$ in an affine manner. Under these assumptions, it is possible to characterize the robust feasible set of many well-known classes of optimization problems in a finite way. For instance, consider the robust linear programming model proposed by Ben-Tal and Nemirovskii [32]

$$
\begin{array}{cl}
\operatorname{minimize} & \max _{\|\Delta c\| \leq \epsilon_{0}}(c+\Delta c)^{T} x \\
\text { subject to } & \left(a_{i}+\Delta a_{i}\right)^{T} x \geq\left(b_{i}+\Delta b_{i}\right), \\
& \text { for all }\left\|\left(\Delta a_{i}, \Delta b_{i}\right)\right\| \leq \epsilon_{i}, \forall i
\end{array}
$$

where each $\epsilon_{i}>0$ is a prespecified scalar. In the above formulation, we have $\delta_{i}=\left(\Delta a_{i}, \Delta b_{i}\right)$ and $\Delta_{i}=$ $\left\{\left(\Delta a_{i}, \Delta b_{i}\right) \mid\left\|\left(\Delta a_{i}, \Delta b_{i}\right)\right\| \leq \epsilon_{i}\right\}$. The main observation is that the robust constraint

$$
\left(a_{i}+\Delta a_{i}\right)^{T} x \geq\left(b_{i}+\Delta b_{i}\right), \quad \text { for all }\left\|\left(\Delta a_{i}, \Delta b_{i}\right)\right\| \leq \epsilon_{i}
$$

is equivalent to the following second-order cone constraint

$$
a_{i}^{T} x-b_{i} \geq \epsilon_{i} \sqrt{1+\|x\|^{2}}
$$

In this way, the robust linear program (56) is reformulated as an equivalent SOCP

$$
\begin{array}{ll}
\text { minimize } & t \\
\text { subject to } & a_{i}^{T} x-b_{i} \geq \epsilon_{i} \sqrt{1+\|x\|^{2}}, \quad i=1,2, \ldots, m, \\
& c^{T} x+\epsilon_{0}\|x\| \leq t .
\end{array}
$$

References [31]-[33] have shown that the robust counterpart of some other well-known convex optimization problems can also be reformulated in a finite way as a conic optimization problem, often as an SOCP or SDP.

As an application example, we consider the robust beamforming problem of finding a $w \in \mathbb{C}^{n}$ such that

$$
\begin{array}{ll}
\text { minimize } & w^{H} R w \\
\text { subject to } & \left|a^{H} w\right| \geq 1, \quad \text { for all }|a-\bar{a}| \leq \epsilon
\end{array}
$$

where $\bar{a}$ is the nominal steering vector, $R$ is the sample correlation matrix, and $\epsilon$ represents the error size in the estimation of steering vector $\bar{a}$. As it stands, the above robust beamforming formulation has an infinitely many nonconvex quadratic constraints. Interestingly, by exploiting the phase ambiguity in $w$ and using the same transformation outlined above, we can show [34] that (57) is equivalent to the following convex optimization problem (SOCP):

$$
\begin{array}{ll}
\operatorname{minimize} & w^{H} R w \\
\text { subject to } & \bar{a}^{H} w \geq 1+\epsilon\|w\|, \operatorname{Im}\left(\bar{a}^{H} w\right)=0
\end{array}
$$

which can be solved efficiently with $O\left(n^{3.5}\right)$ complexity.

\section{CONCLUDING REMARKS}

Convex optimization provides a powerful set of tools for the design and analysis of communication systems and signal processing algorithms. Convex optimization techniques are useful both in obtaining structural insights to the optimal solution, as well as in providing provably optimal numerical solutions to the problem efficiently. This tutorial contains only a small sample of recent applications of convex optimization in communications and signal processing. Future applications of these techniques will likely yield many more interesting results.

\section{REFERENCES}

[1] Y. Nesterov and A. Nemirovskii, Interior Point Polynomial Methods in Convex Programming. Philadelphia, PA: SIAM, 1994, vol. 13, Studies in Applied Mathematics.

[2] H. Wolkowicz, R. Saigal, and L. Vandenberghe, Handbook of Semidefinite Programming: Theory, Algorithms and Applications. Norwell, MA: Kluwer, 1999

[3] J. F. Sturm, "Using SeDuMi 1.02, a Matlab toolbox for optimization over symmetric cones," Optim. Methods Softw. vol. 11-12, pp. 625-653, 1999. [Online]. Available: http://www.fewcal.kub.nl/sturm/ software/sedumi.html

[4] M. Chiang, "Geometric programming for communication systems," Foundations and Trends Commun. Inf. Theory, vol. 2, no. 1-2, pp. 1-156, Aug. 2005.

[5] S. Boyd and L. Vandenberghe, Convex Optimization. Cambridge, U.K.: Cambridge Univ. Press, 2004.

[6] F. Rashid-Farrokhi, K. J. R. Liu, and L. Tassiulas, "Transmit beamforming and power control for cellular wireless systems," IEEE J. Sel. Areas Commun., vol. 16, no. 8, pp. 1437-1450, Oct. 1998.

[7] E. Visotsky and U. Madhow, "Optimum beamforming using transmit antenna arrays," in Proc. IEEE Veh. Technol. Conf., May 1999, vol. 1, pp. 851-856.

[8] F. Rashid-Farrokhi, L. Tassiulas, and K. J. R. Liu, "Joint optimal power control and beamforming in wireless networks using antenna arrays," IEEE J. Sel. Areas Commun., vol. 46, no. 10, pp. 1313-1324, Oct. 1998.

[9] M. Schubert and H. Boche, "Solution of the multiuser downlink beamforming problem with individual SINR constraints," IEEE Trans. Veh. Technol., vol. 53, no. 1, pp. 18-28, Jan. 2004.

[10] M. Bengtsson and B. Ottersten, "Optimal and suboptimal transmit beamforming," in Handbook of Antennas in Wireless Communications, L. C. Godara, Ed. Boca Raton, FL: CRC, 2002.

[11] A. Wiesel, Y. C. Eldar, and S. Shamai, "Linear precoding via conic optimization for fixed MIMO receivers," IEEE Trans. Signal Process., vol. 54, no. 3, pp. 161-176, Jan. 2006.

[12] S. Vishwanath, N. Jindal, and A. Goldsmith, "Duality, achievable rates, and sum-rate capacity of Gaussian MIMO broadcast channels," IEEE Trans. Inf. Theory, vol. 49, no. 10, pp. 2658-2668, Oct. 2003.

[13] P. Viswanath and D. Tse, "Sum capacity of the multiple antenna Gaussian broadcast channel and uplink-downlink duality," IEEE Trans. Inf. Theory, vol. 49, no. 8, pp. 1912-1921, Aug. 2003.

[14] M. Schubert and H. Boche, "Iterative multiuser uplink and downlink beamforming under SINR constraints," IEEE Trans. Signal Process., vol. 53, no. 7, pp. 2324-2334, Jul. 2005.

[15] G. Caire and S. Shamai, "On the achievable throughput of a multiantenna Gaussian broadcast channel," IEEE Trans. Inf. Theory, vol. 49, pp. 1691-1706, Jul. 2003.

[16] H. Weingarten, Y. Steinberg, and S. Shamai, "Capacity region of the Gaussian MIMO broadcast channel," IEEE Trans. Inf. Theory, 2006, to be published.

[17] W. Yu and T. Lan, "Transmitter optimization for the multi-antenna downlink with per-antenna power constraints," IEEE Trans. Signal Process., 2005, submitted for publication.

[18] N. Jindal, W. Rhee, S. Vishwanath, S. A. Jafar, and A. Goldsmith, "Sum power iterative water-filling for multi-antenna Gaussian broadcast channels," IEEE Trans. Inf. Theory, vol. 51, no. 4, pp. 1570-1580, Apr. 2005.

[19] W. Yu, "Sum-capacity computation for the Gaussian vector broadcast channel via dual decomposition," IEEE Trans. Inf. Theory, vol. 52, no. 2, pp. 754-759, Feb. 2006. 
[20] — - "Uplink-downlink duality via minimax duality," IEEE Trans. Inf. Theory, vol. 52, no. 2, pp. 361-374, Feb. 2006.

[21] B. Song, R. L. Cruz, and B. D. Rao, "Network duality and its application to multi-user MIMO wireless networks with SINR constraints," in Proc. IEEE Int. Conf. Commun., May 2005, vol. 4, pp. 2684-2689.

[22] R. Cendrillon, W. Yu, M. Moonen, J. Verliden, and T. Bostoen, "Optimal spectrum balancing for digital subscriber lines," IEEE Trans. Commun., vol. 54, no. 5, pp. 922-933, May 2006.

[23] W. Yu and R. Lui, "Dual methods for nonconvex spectrum optimization of multicarrier systems," IEEE Trans. Commun., vol. 54, no. 7, Jul. 2006.

[24] J. Papandriopoulos and J. Evans, "Low-complexity distributed algorithms for spectrum balancing in multi-user DSL networks," in Proc. IEEE Int. Conf. Commun., Jun. 2006.

[25] M. Chiang, C. W. Tan, D. Palomar, D. O'Neil, and D. Julian, "Power control by geometric programming," IEEE Trans. Wireless Commun., submitted for publication, 2005, Appeared in part at IEEE INFOCOM, New York, Jun. 2002.

[26] U. Fincke and M. Pohst, "Improved methods for calculating vectors of short length in a lattice, including a complexity analysis," Math. Comput., vol. 44, no. 1, pp. 463-471, Apr. 1985.

[27] J. Jaldén and B. Ottersten, "On the complexity of sphere decoding in digital communications," IEEE Trans. Signal Process., vol. 53, no. 4, pp. 1474-1484, Apr. 2005.

[28] M. Kisialiou and Z.-Q. Luo, "Performance analysis of quasi-maximum-likelihood detector based on semi-definite programming," in Proc. IEEE Int. Conf. Acoust., Speech, Signal Process., 2005, vol. 3, pp. 433-436.

[29] N. D. Sidiropoulos, T. N. Davidson, and Z.-Q. Luo, "Transmit beamforming for physical layer multicasting," IEEE Trans. Signal Process., vol. 54, no. 6, pp. 2239-2251, Jun. 2006.

[30] Z.-Q. Luo, N. D. Sidiropoulos, P. Tseng, and S. Zhang, "Approximation bounds for quadratic optimization with homogeneous quadratic constraints," SIAM J. Optimization, 2006, accepted for publication.

[31] A. Ben-Tal, L. El Ghaoui, and A. Nemirovskii, "Robust semidefinite programming," in Handbook of Semidefinite Programming, $\mathrm{H}$ Wolkowicz, R. Saigal, and L. Vandenberghe, Eds. Norwell, MA: Kluwer, Mar. 2000.

[32] A. Ben-Tal and A. Nemirovskii, "Robust convex optimization," Math. Oper. Res., vol. 23, pp. 769-805, 1998.

[33] L. El Ghaoui, F. Oustry, and H. Lebret, "Robust solutions to uncertain semidefinite programs," SIAM J. Optimization, vol. 9, no. 1, pp. 33-52, 1998.

[34] S. Vorobyov, A. Gershman, and Z.-Q. Luo, "Robust adaptive beamforming using worst-case performance optimization: a solution to the signal mismatch problem," IEEE Trans. Signal Process., vol. 51, pp. 313-323, 2003.

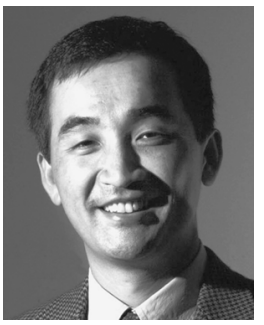

Zhi-Quan (Tom) Luo (SM'03) received the B.Sc. degree in mathematics from Peking University, Peking, China, in 1984. From 1985 to 1989, he studied at the Operations Research Center and the Department of Electrical Engineering and Computer Science, Massachusetts Institute of Technology, Cambridge, where he received the Ph.D. degree in operations research.

During the academic year of 1984 to 1985 , he was with the Nankai Institute of Mathematics, Tianjin, China. In 1989, he joined the Department of Electrical and Computer Engineering, McMaster University, Hamilton, ON, Canada, where he became a Professor in 1998 and held the Canada Research Chair in Information Processing since 2001. Since April 2003, he has been a Professor with the Department of Electrical and Computer Engineering and holds an endowed ADC Research Chair in Wireless Telecommunications with the Digital Technology Center, University of Minnesota. His research interests lie in the union of large-scale optimization, information theory and coding, data communications, and signal processing.

Prof. Luo received an IEEE Signal Processing Society Best Paper Award in 2004. He is a member of the Society for Industrial and Applied Mathematics (SIAM) and MPS. He is also a member of the Signal Processing for Communications (SPCOM) and Signal Processing Theory and Methods (SPTM) Technical Committees of the IEEE Signal Processing Society. From 2000 to 2004, he served as an Associate Editor for the IEEE TRANSACTIONS ON SignaL PROCESSING and Mathematics of Computation. He is presently serving as an Associate Editor for several international journals including SIAM Journal on Optimization and Mathematics of Operations Research.

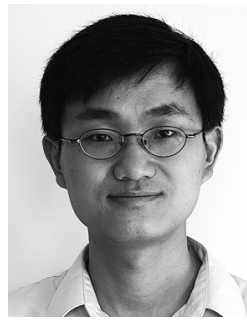

Wei Yu (S'97-M'02) received the B.A.Sc. degree in computer engineering and mathematics from the University of Waterloo, Waterloo, ON, Canada, in 1997, and the M.S. and Ph.D. degrees in electrical engineering from Stanford University, Stanford, CA, in 1998 and 2002, respectively.

Since 2002, he has been an Assistant Professor with the Electrical and Computer Engineering Department, University of Toronto, Toronto, ON, Canada, where he also holds a Canada Research Chair. His main research interests are multiuser information theory, optimization, wireless communications, and broadband access networks.

Prof. $\mathrm{Yu}$ is an Editor of the IEEE TRANSACTIONS ON Wireless COMMUNICATIONS and a Guest Editor of the EURASIP Journal on Applied Signal Processing (Special Issue on Advanced Signal Processing for Digital Subscriber Lines). 